\title{
THE CFL CONDITION FOR SPECTRAL APPROXIMATIONS TO HYPERBOLIC INITIAL-BOUNDARY VALUE PROBLEMS
}

\author{
DAVID GOTTLIEB AND EITAN TADMOR
}

\begin{abstract}
We study the stability of spectral approximations to scalar hyperbolic initial-boundary value problems with variable coefficients. Time is discretized by explicit multi-level or Runge-Kutta methods of order $\leq 3$ (forward Euler time-differencing is included), and we study spatial discretizations by spectral and pseudospectral approximations associated with the general family of Jacobi polynomials. We prove that these fully explicit spectral approximations are stable provided their time step, $\Delta t$, is restricted by the CFL-like condition $\Delta t<$ Const $\cdot N^{-2}$, where $N$ equals the spatial number of degrees of frecdom. We give two independent proofs of this result, depending on two different choices of appropriate $L^{2}$-weighted norms. In both approaches, the proofs hinge on a certain inverse inequality interesting for its own sake. Our result confirms the commonly held belief that the above CFL stability restriction, which is extensively used in practical implementations, guarantees the stability (and hence the convergence) of fully-explicit spectral approximations in the nonperiodic case.
\end{abstract}

\section{INTRODUCTION}

We are concerned here with fully discrete spectral and pseudospectral approximations to scalar hyperbolic equations. In this context, the spectral (and, respectively, the pseudospectral) approximations consist of truncation (and, respectively, collocation) of $N$-term spatial expansions, which are expressed in terms of general Jacobi polynomials; Chebyshev and Legendre expansions are the ones most frequently found in practice. In this paper we prove that such $N$-term approximations are stable, provided their time step, $\Delta t$, fulfills the CFL-like condition $\Delta t \leq$ Const $\cdot N^{-2}$. To clarify the origin of such a CFL-like condition in our case, we recall that the Jacobi polynomials are in fact the eigenfunctions of second-order singular Sturm-Liouville problems. Our arguments show that the main reason for the above CFL limitation is the $O\left(N^{2}\right)$ growth of the $N$ th eigenvalue associated with these Sturm-Liouville problems.

Received April 6, 1990; revised June 21, 1990.

1980 Mathematics Subject Classification (1985 Revision). Primary 65M10, 65N35.

Research was supported in part by NASA Contract No. NAS1-18605 while both authors were in residence at the Institute for Computer Applications in Science and Engineering (ICASE), NASA Langley Research Center, Hampton, VA 23665. The first author was also supported by the Air Force Office of Scientific Research grant no. AFOSR-90-0093, by DARPA-URI contract N0001486-k0754, and by NSF grant DMS-88-10150. 
The paper is organized as follows. Section 2 includes a brief summary on the properties of Jacobi polynomials (and their quadrature rules), which are used throughout the paper. In $\S 3$ we state our main stability theorems for forward Euler time-differencing and (pseudo)spectral spatial differencing, for constantcoefficient equations with homogeneous boundary conditions. Section 4 extends our stability results to the inhomogeneous case. In $\S 5$ we discuss multi-level and Runge-Kutta time-differencing. Finally, in $\S 6$ we show how to extend our results in the presence of (positive) variable coefficients.

\section{VERY SHORT GUIDE TO JACOBI POLYNOMIALS}

Jacobi polynomials, $P_{k}^{(\alpha, \beta)}$, are the eigenfunctions of the singular SturmLiouville problem

$$
\left(\left(1-x^{2}\right) w(x) P_{k}^{(\alpha, \beta)^{\prime}}(x)\right)^{\prime}+\lambda_{k} w(x) P_{k}^{(\alpha, \beta)}(x)=0, \quad-1 \leq x \leq 1,
$$

with corresponding eigenvalues $\lambda_{k}$,

$$
\lambda_{k} \equiv \lambda_{k}(\alpha, \beta)=k(k+\alpha+\beta+1) .
$$

Different families of Jacobi polynomials are associated with different weight functions $w(x)$,

$$
w(x) \equiv w(x ; \alpha, \beta)=(1-x)^{\alpha}(1+x)^{\beta}, \quad \alpha, \beta>-1 .
$$

In the sequel we shall frequently use several properties of the Jacobi polynomials. A brief summary of these properties is given below (consult, e.g., [13]). We start with the well-known

Property 1 (Orthogonality). We have

$$
\left(P_{j}^{(\alpha, \beta)}, P_{k}^{(\alpha, \beta)}\right)_{w(x)}=0, \quad j \neq k .
$$

The derivatives of Jacobi polynomials are also Jacobi polynomials. This is evident from the following property, which shows that $\left\{P_{k}^{(\alpha, \beta)^{\prime}}\right\}_{k \geq 0}$ are orthogonal with respect to the weight $\left(1-x^{2}\right) w(x) \equiv w(x ; \alpha+1, \beta+1)$ :

$$
P_{k+1}^{(\alpha, \beta)^{\prime}}=\text { Const }_{k, \alpha, \beta} P_{k}^{(\alpha+1, \beta+1)}, \quad \text { Const }_{k, \alpha, \beta}=\frac{1}{2}(k+\alpha+\beta+2) .
$$

Property 2 (Orthogonality of derivatives). We have

$$
\begin{gathered}
\left(P_{j}^{(\alpha, \beta)^{\prime}}, P_{k}^{(\alpha, \beta)^{\prime}}\right)_{\left(1-x^{2}\right) w(x)}=0, \quad j \neq k, \\
\left\|P_{k}^{(\alpha, \beta)^{\prime}}\right\|_{\left(1-x^{2}\right) w(x)}^{2}=\lambda_{k}\left\|P_{k}^{(\alpha, \beta)}\right\|_{w(x)}^{2} .
\end{gathered}
$$

Indeed, equalities (2.4) and (2.5) follow from integration by parts of $(2.1)$ against $P_{j}^{(\alpha, \beta)}(x)$. 
Let $\pi_{N}$ denote the space of algebraic polynomials with degree $\leq N$. A useful consequence of the last two properties is provided by

Lemma 2.1. (Inverse inequality). For all $p \in \pi_{N}$ we have

$$
\left\|p^{\prime}\right\|_{\left(1-x^{2}\right) \omega(x)} \leq \sqrt{\lambda_{N}}\|p\|_{\omega(x)}, \quad p \in \pi_{N} .
$$

Here, $\omega(x)$ stands for an arbitrary $w(x ; \alpha, \beta)$-weight, and $\lambda_{N} \equiv \lambda_{N}(\alpha, \beta)$ is the corresponding Nth eigenvalue.

Remarks. 1. Inequality (2.6) can be viewed as the algebraic analogue of the trigonometric inverse inequality,

$$
\begin{aligned}
& \left\|p^{\prime}\right\|_{L^{2}[-\pi, \pi]} \leq N\|p\|_{L^{2}[-\pi, \pi]}, \\
& \quad p=\text { any trigonometric polynomial of order } N .
\end{aligned}
$$

This should be contrasted with a similar $L^{2}$-inverse inequality for algebraic polynomials, where there is a loss of an $N^{2}$-factor for each derivative [3],

$$
\begin{aligned}
& \left\|p^{\prime}\right\|_{L^{2}[-1,1]} \leq \text { Const } \cdot N^{2}\|p\|_{L^{2}[-1,1]}, \\
& \qquad p=\text { any algebraic polynomial of degree } N,
\end{aligned}
$$

and this estimate, $(2.7 \mathrm{~b})$, is sharp in view of, e.g., the $\pi_{N}$-polynomial $p_{N}(x)=$ $\sum_{k=0}^{N} P_{k}^{(-1 / 2,-1 / 2)}(x)$. Thus, the use of the differently weighted $L^{2}$-norms in the algebraic case, (2.6), is essential in order to retain a loss of only a $\sqrt{\lambda_{N}} \sim N$ factor for each derivative.

2. The inverse inequality (2.6) can be viewed as an $L^{2}$-weighted version of Bernstein's inequality

$$
\left\|\left(1-x^{2}\right)^{1 / 2} p^{\prime}(x)\right\|_{L^{\infty}[-1,1]} \leq N\|p(x)\|_{L^{\infty}[-1,1]}, \quad p \in \pi_{N} .
$$

Standard interpolation arguments between this $L^{\infty}$-type estimate and the $L^{2}$ type estimate (2.6) yield for $q \geq 2$

$$
\begin{aligned}
& \left\|\left(1-x^{2}\right)^{1 / 2} p^{\prime}(x)\right\|_{L_{w(x ; \alpha, \beta)}^{q}[-1,1]} \\
& \quad \leq \sqrt{\lambda_{N}(\alpha, \beta)} \cdot\|p(x)\|_{L_{w(x ; \alpha, \beta)}^{q}[-1,1]}, \quad p \in \pi_{N} .
\end{aligned}
$$

Similar weighted $L^{q}$-type estimates apply to higher derivatives.

Proof of Lemma 2.1. Given $p(x)$ in $\pi_{N}$, we will use its Jacobi expansion, $p(x)=\sum_{k=0}^{N} a_{k} P_{k}^{(\alpha, \beta)}(x)$ and $p^{\prime}(x)=\sum_{k=0}^{N} a_{k} P_{k}^{(\alpha, \beta)^{\prime}}(x)$. Starting with the left-hand side of (2.6) and using (2.4), (2.5), and (2.2) in this order, we obtain

$$
(\mathrm{LHS})^{2}=\sum_{k=0}^{N} a_{k}^{2}\left\|P_{k}^{(\alpha, \beta)^{\prime}}\right\|_{\left(1-x^{2}\right) w(x)}^{2}=\sum_{k=0}^{N} \lambda_{k} a_{k}^{2}\left\|P_{k}^{(\alpha, \beta)}\right\|_{w(x)}^{2} \leq(\mathrm{RHS})^{2} .
$$


We note in passing that Lemma 2.1 can be generalized to higher derivatives: successive application of $(2.6)$ with $\omega(x)=w(x ; \alpha, \beta)$ yields

$$
\left\|p^{(k)}(x)\right\|_{\left(1-x^{2}\right)^{k} w(x)}^{2} \leq \prod_{j=0}^{k-1} \lambda_{N}(\alpha+j, \beta+j) \cdot\|p(x)\|_{w(x)}^{2}, \quad p \in \pi_{N} .
$$

This leads us to a "natural" definition of nonperiodic Sobolev spaces equipped with finite $H_{w(x)}^{s}$-norm, where

$$
\|p\|_{H_{w(x)}^{s}}^{2}=\sum_{k=0}^{s}\left\|p^{(k)}\right\|_{\left(1-x^{2}\right)^{k} w(x)}^{2} .
$$

With this in mind, we now recover a sharp inverse inequality familiar from the trigonometric setup,

$$
\|p\|_{H_{w(x)}^{s}} \leq \text { Const }_{s} \cdot N^{s}\|p\|_{w(x)}, \quad \text { Const } \sim 1+s / N, p \in \pi_{N} .
$$

In the above discussion we can replace integrals by discrete summations, in view of the well-known

Property 3 (Gauss quadrature rule). Let $\left\{q_{N}(x)\right\}_{N \geq 0}$ be a family of $\pi_{N}$-polynomials orthogonal with respect to the $\omega(x)$-weighted $L^{2}$ inner product. Let $-1<x_{1}<x_{2}<\cdots<x_{N}<1$ be the $N$ zeros of $q_{N}(x)$. Then there exist positive weights, $\left\{\omega_{j}\right\}_{j=1}^{N}$, such that for all $p \in \pi_{2 N-1}$ we have

$$
\int_{-1}^{1} \omega(x) p(x) d x=\sum_{j=1}^{N} \omega_{j} p\left(x_{j}\right), \quad p \in \pi_{2 N-1} .
$$

Remark. To compute the Gauss weights, we set $p(x)=q_{N}(x) /\left(x-x_{k}\right)$ in (2.11). Since $p\left(x_{j}\right)=0, j \neq k,(2.11)$ yields

$$
\omega_{k}=\frac{1}{q_{N}^{\prime}\left(x_{k}\right)} \int_{-1}^{1} \omega(x) \frac{q_{N}(x)}{x-x_{k}} d x, \quad 1 \leq k \leq N .
$$

Examples. 1. Gauss-Jacobi quadrature rule. By Property 1, (2.11) applies to $\left\{P_{N}^{(\alpha, \beta)}\right\}_{N \geq 1}$ with $\omega(x)=w(x ; \alpha, \beta)$. Hence, there exist $\left\{w_{j}=w_{j}^{G}(\alpha, \beta)\right\}_{j=1}^{N}$ such that

$$
\int_{-1}^{1} w(x) p(x) d x=\sum_{j=1}^{N} w_{j} p\left(x_{j}\right) \text { for all } p \in \pi_{2 N-1} .
$$

Remark. The Gauss-Jacobi quadrature rule (2.13) can be used as a highly accurate quadrature rule for general smooth, not necessarily polynomial functions. The error incurred is governed by $[4, p .75]$

$$
\int_{-1}^{1} w(x) f(x)-\sum_{j=1}^{N} w_{j} f\left(x_{j}\right)=\text { Const } \cdot f^{(2 N)}(\theta)
$$

$$
\text { Const }>0,|\theta| \leq 1 \text {. }
$$

2. Gauss-Lobatto-Jacobi quadrature rule. By Property 2, (2.11) applies to $\left\{P_{N+1}^{(\alpha, \beta)^{\prime}}\right\}_{N \geq 0}$ with $\omega(x)=\left(1-x^{2}\right) w(x ; \alpha, \beta)$, and therefore, there exist 
$\left\{\tilde{w}_{j}=w_{j}^{G}(\alpha+1, \beta+1)\right\}_{j=1}^{N}$ such that

$$
\int_{-1}^{1}\left(1-x^{2}\right) w(x) r(x) d x=\sum_{j=1}^{N} \tilde{w}_{j} r\left(x_{j}\right) \text { for all } r \in \pi_{2 N-1} .
$$

This is in fact a special case of the Gauss-Lobatto-Jacobi quadrature rule which is exact for all $p \in \pi_{2 N+1}$. Indeed, for all such $p$ 's we have $p(x)=$ $\left(1-x^{2}\right) r(x)+l(x)$ with $r(x)$ in $\pi_{2 N-1}$ and a linear $l(x)=p(-1) \frac{1-x}{2}+p(1) \frac{1+x}{2}$. By (2.15),

$$
\begin{aligned}
\int_{-1}^{1} w(x) p(x) d x & =\sum_{j=1}^{N} \tilde{w}_{j} r\left(x_{j}\right)+\int_{-1}^{1} w(x) l(x) \\
& =\sum_{j=1}^{N} \frac{\tilde{w}_{j}}{1-x_{j}^{2}} p\left(x_{j}\right)+\int_{-1}^{1} w(x) l(x)-\sum_{j=1}^{N} \frac{\tilde{w}_{j}}{1-x_{j}^{2}} l\left(x_{j}\right) \\
& =\mathrm{I}+\mathrm{II}+\mathrm{III} .
\end{aligned}
$$

Thus, we have

$$
\mathrm{I}=\sum_{j=1}^{N} w_{j}^{L} p\left(x_{j}\right), \quad w_{j}^{L} \equiv \frac{\tilde{w}_{j}}{1-x_{j}^{2}}=\frac{1}{1-x_{j}^{2}} w_{j}^{G}(\alpha+1, \beta+1),
$$

and the two expressions, II + III, amount to a linear combination of $p(-1)$ and $p(1)$,

$$
\mathrm{II}+\mathrm{III}=w_{0}^{L} p\left(x_{0}\right)+w_{N+1}^{L} p\left(x_{N+1}\right), \quad x_{0} \equiv-1<x_{1}<\cdots<x_{N}<1 \equiv x_{N+1} .
$$

Hence, there exist $\left\{w_{j}=w_{j}^{L}(\alpha, \beta)\right\}_{j=0}^{N+1}$ such that

$$
\int_{-1}^{1} w(x) p(x) d x=\sum_{j=0}^{N+1} w_{j} p\left(x_{j}\right) \text { for all } p \in \pi_{2 N+1}
$$

Finally, we shall need some information on the behavior of the collocation points which appear on the right of (2.13) and (2.16). To this end, we quote [13, Theorem 8.1.2] as

Property 4 (Distribution of zeros). If $x_{j}=\cos \theta_{j}$ is the jth zero of $P_{N}^{(\alpha, \beta)}(x)$, then for fixed $j, N \theta_{j}$ is asymptotically equal to the jth zero of the Bessel function of order $\alpha$, and hence

$$
\begin{aligned}
& 1-x_{j}^{2}=\sin ^{2} \theta_{j} \sim \text { Const }_{j_{0}} N^{-2} \\
& \qquad \text { for } j \in J=\left\{1 \leq j \leq j_{0}, N-j_{0} \leq j \leq N\right\} .
\end{aligned}
$$

Thus, the zeros of $P_{N}^{(\alpha, \beta)}(x)$ accumulate within $O\left(N^{-2}\right)$-neighborhoods of $\{-1,+1\}$. For example, more concrete estimates, e.g., [13, Theorem 6.21.2], yield the upper bound

$$
\frac{1}{1-x_{j}} \leq \frac{1}{2}\left(N+\frac{1}{2}\right)^{2}, \quad 1 \leq j \leq N, \alpha, \beta \in\left[-\frac{1}{2}, \frac{1}{2}\right] .
$$




\section{FORWARD EULER WITH HOMOGENEOUS BOUNDARY CONDITIONS}

We start with the scalar constant-coefficient hyperbolic equation,

$$
u_{t}=a u_{x}, \quad(x, t) \in[-1,1] \times[0, \infty), a>0,
$$

which is augmented with homogeneous conditions at the inflow boundary,

$$
u(1, t)=0, \quad t>0 .
$$

To approximate (3.1), we use forward Euler time-differencing on the left, and either spectral or pseudospectral differencing on the right. Thus, we seek a temporal sequence of spatial $\pi_{N}$-polynomials, $v^{m}=v_{N}\left(x, t^{m}=m \Delta t\right)$, such that

$$
v_{N}\left(x, t^{m}+\Delta t\right)=v_{N}\left(x, t^{m}\right)+\Delta t \cdot a v_{N}^{\prime}\left(x, t^{m}\right)+\Delta t \cdot \tau\left(t^{m}\right) q_{N}(x) .
$$

Here, $q_{N}(x)$ is a $\pi_{N}$-polynomial which characterizes the specific (pseudo)spectral method we employ, and $\tau=\tau\left(t^{m}\right)$ is a free scalar multiplier to be determined by the boundary constraint

$$
v_{N}\left(x=1, t^{m}\right)=0 .
$$

We shall study the spectral tau methods $[8,2]$ associated with Jacobi polynomials $P_{N}^{(\alpha, \beta)}(x), \alpha, \beta \in(-1,1)$,

$$
\begin{aligned}
v_{N}\left(x, t^{m}+\Delta t\right) & =v_{N}\left(x, t^{m}\right)+\Delta t \cdot a v_{N}^{\prime}\left(x, t^{m}\right)+\Delta t \cdot \tau\left(t^{m}\right) q_{N}(x), \\
q_{N}(x) & =P_{N}^{(\alpha, \beta)}(x),
\end{aligned}
$$

and the pseudospectral Jacobi methods [5, 2], which are collocated at the interior extrema of $P_{N+1}^{(\alpha, \beta)}, \alpha, \beta \in(-1,0)$, i.e.,

$$
\begin{aligned}
v_{N}\left(x, t^{m}+\Delta t\right) & =v_{N}\left(x, t^{m}\right)+\Delta t \cdot a v_{N}^{\prime}\left(x, t^{m}\right)+\Delta t \cdot \tau\left(t^{m}\right) q_{N}(x), \\
q_{N}(x) & =P_{N+1}^{(\alpha, \beta)^{\prime}}(x) .
\end{aligned}
$$

Remark. These two families of spectral and pseudospectral Jacobi methods are closely related since $P_{N+1}^{(\alpha, \beta)^{\prime}}(x)$ is a scalar multiple of $P_{N}^{(\alpha+1, \beta+1)}(x)$ (consult (2.3)). We will not discuss here a different alternative to (3.5), where one collocates at the interior extrema of $P_{N}^{(\alpha, \beta)}(x)$ together with the downstream outflow boundary, so that

$$
q_{N}(x)=(1+x) P_{N}^{(\alpha, \beta)^{\prime}}(x) .
$$

Let $-1<x_{1}<x_{2}<\cdots<x_{N}<1$ be the $N$ distinct zeros of the forcing polynomial $q_{N}(x)$. The spectral approximation (3.3a) restricted to these points reads

$$
v_{N}\left(x_{j}, t^{m+1}\right)=v_{N}\left(x_{j}, t^{m}\right)+\Delta t \cdot a v_{N}^{\prime}\left(x_{j}, t^{m}\right), \quad 1 \leq j \leq N,
$$

and is augmented with the homogeneous boundary conditions

$$
v_{N}\left(1, t^{m}\right)=0 \text {. }
$$

Equations (3.7a), (3.7b) furnish a complete equivalent formulation of the spectral approximation $(3.3 \mathrm{a}),(3.3 \mathrm{~b})$. An essential ingredient in a stability theory 
of such approximations lies in the choice of appropriate $L^{2}$-weighted norms

$$
\|f(x)\|_{\omega}^{2}=\langle f(x), f(x)\rangle, \quad\langle f(x), g(x)\rangle=\sum_{j=1}^{N} \omega_{j} f\left(x_{j}\right) g\left(x_{j}\right) .
$$

We make

Definition 3.1 (Stability). The approximation (3.7a), (3.7b) is stable if there exist discrete weights, $\left\{\omega_{j}>0\right\}_{j=1}^{N}$, and a constant $\eta_{0}$ independent of $N$, such that

$$
\left\|v_{N}(\cdot, t)\right\|_{\omega} \leq \text { Const } \cdot e^{\eta_{0} t}\left\|v_{N}(\cdot, 0)\right\|_{\omega} .
$$

The approximation (3.7a), (3.7b) is strongly stable if (3.9) holds with Const $=1$ and $\eta_{0} \leq 0$, i.e., if

$$
\left\|v_{N}(\cdot, t)\right\|_{\omega} \leq\left\|v_{N}(\cdot, 0)\right\|_{\omega}
$$

We recall that in the Jacobi-type spectral approximations (3.4) and (3.5), the nodes $\left\{x_{j}\right\}_{j=1}^{N}$ are the zeros of Jacobi polynomials associated with the Gauss and Gauss-Lobatto quadrature rules. We use

$$
\Delta x_{\min }=\min \left(1+x_{1}, 1-x_{N}\right)
$$

to measure the minimal grid size associated with these Gauss nodes. Our choice of discrete weights $\left\{\omega_{j}\right\}_{j=1}^{N}$ for the stability of the spectral and pseudospectral Jacobi methods (3.4), (3.5) will be specified later on; these weights are related (but not equal) to the corresponding Gauss weights $\left\{w_{j}\right\}_{j=1}^{N}$ indicated earlier.

With this in mind, we have

Theorem 3.1 (Stability of the spectral and pseudospectral Jacobi methods). Consider the spectral approximations (3.7a), (3.7b), associated with the Jacobi tau method (3.4), or the pseudospectral Jacobi method (3.5). There exists a positive constant $\eta_{0} \equiv \eta_{0}(\alpha, \beta)>0$ independent of $N$ such that if the following $C F L$ condition holds:

$$
\Delta t \cdot a\left(\lambda_{N-1}+\frac{2}{\Delta x_{\min }}\right) \leq \eta_{0}
$$

then the approximation $(3.7 \mathrm{a}),(3.7 \mathrm{~b})$ is strongly stable, and the following estimate is fulfilled:

$$
\left\|v_{N}(\cdot, t)\right\|_{\omega} \leq e^{-\eta_{0} a t}\left\|v_{N}(\cdot, 0)\right\|_{\omega} .
$$

Notes. 1. The choice of $L^{2}$-weighted norms. Theorem 3.1 deals with the stability of both the spectral tau methods associated with Jacobi polynomials $P_{N}^{(\alpha, \beta)}(x)$, $\alpha, \beta \in(-1,1)$, and the closely related pseudospectral methods associated with $P_{N+1}^{(\alpha, \beta)^{\prime}}(x), \alpha, \beta \in(-1,0)$. In each case we give two different stability proofs, which are based on two different choices of discrete $L^{2}$-weighted norms; these 
discrete weights $\left\{\omega_{j}\right\}_{j=1}^{N}$ are given by

$\begin{array}{rlrl}\text { (3.14a) } \omega_{j}=\frac{1+x_{j}}{1-x_{j}} w_{j}, & \left\{w_{j}\right\}_{j=1}^{N}=\text { Gauss-Jacobi weights in (2.13), } \\ \text { (3.14b) } \omega_{j}=\left(1+x_{j}\right) w_{j}, & \left\{w_{j}\right\}_{j=1}^{N}=\text { (interior) Gauss-Lobatto-Jacobi } \\ \text { weights in }(2.16) .\end{array}$

2. The CFL condition. The CFL condition (3.12) places an $O\left(N^{-2}\right)$ stability restriction on the time step $\Delta t$, and this stability restriction involves two factors. First, since we expand our solution in terms of the eigenfunctions of the SturmLiouville problem (2.1), the CFL condition involves the corresponding $(N-1)$ st eigenvalue

$$
\lambda_{N-1} \equiv \lambda_{N-1}(\alpha, \beta)<(N+1)^{2}, \quad \alpha, \beta \in(-1,1) .
$$

Second, the spectral Jacobi approximation (3.7a) is collocated at Gauss nodes, which accumulate within $O\left(N^{-2}\right)$ neighborhoods near the boundaries, i.e., by (2.17a),

$$
\frac{1}{\Delta x_{\min }} \leq \text { Const } \cdot N^{2} \text {. }
$$

Thus, in view of (3.15a), (3.15b), the CFL condition (3.12) boils down to

$$
\Delta t \cdot a N^{2} \leq \text { Const }_{\alpha, \beta} \text {. }
$$

In particular, for the practical range of parameters, $\alpha, \beta \in\left[-\frac{1}{2}, \frac{1}{2}\right],(2.17 \mathrm{~b})$ implies that Const C, $\beta \sim \frac{1}{2} \eta_{0}(\alpha, \beta)$.

3. The choice of a stability norm. The stability statement asserted in Theorem 3.1 is formulated in terms of discrete seminorms, $\|\cdot\|_{\omega}$, which are $\omega$-weighted by either (3.14a) or (3.14b). We note that $\|\cdot\|_{\omega}$ are in fact well defined norms on the space of $\pi_{N}$-polynomials satisfying the vanishing boundary condition (3.7b), i.e., corresponding to (3.14a) or (3.14b) we have in view of $(2.14)$,

$$
\left\|v_{N}(\cdot, t)\right\|_{\omega} \geq \int_{-1}^{1} w(x) \frac{1+x}{1-x} v_{N}^{2}(x, t) d x, \quad v_{N}(1, t)=0,
$$

and in view of $(2.16)$,

$$
\left\|v_{N}(\cdot, t)\right\|_{\omega}=\int_{-1}^{1} w(x)(1+x) v_{N}^{2}(x, t) d x, \quad v_{N}(1, t)=0
$$

Moreover, in view of (3.15b), one may convert the stability statement (3.13) into the usual $L_{2}$-type stability estimate at the expense of possible algebraic growth, which reads

$$
\begin{gathered}
\left\|v_{N}(\cdot, t)\right\|_{w(x)} \leq \text { Const } \cdot N^{2} e^{-\eta_{0} a t}\left\|v_{N}(\cdot, 0)\right\|_{w(x)}, \\
\left\|v_{N}(\cdot, t)\right\|_{w(x)}^{2}=\int_{-1}^{1} w(x) v_{N}^{2}(x, t) d x
\end{gathered}
$$


4. Exponential time decay. Let us integrate by parts the differential equation (3.1) against $(1+x) u$. Thanks to the homogeneous boundary condition (3.2) we find

$$
\frac{d}{d t} \int_{-1}^{1}(1+x) u^{2}(x, t) d x \leq-\frac{a}{2} \int_{-1}^{1}(1+x) u^{2}(x, t) d x
$$

and therefore,

$$
\|u(\cdot, t)\|_{1+x} \leq e^{-a t / 4}\|u(\cdot, 0)\|_{1+x} .
$$

This estimate corresponds to the special case of the stability statement (3.13) for the spectral Legendre tau method $(\alpha=\beta=0)$ weighted by $(3.14 \mathrm{~b})$. The exponential time decay indicated in (3.20), and more generally in (3.13), is due to the special choice of $\omega$-weighted stability norms. The weights $\left\{w_{j}\right\}_{j=1}^{N}$ in $(3.14 a),(3.14 b)$ involve the essential factors $1+x_{j}$ or $\left(1+x_{j}\right) /\left(1-x_{j}\right)$ which amplify the inflow boundary values in comparison to the outflow ones. Since in the current homogeneous case, vanishing inflow data is propagating into the domain, this results in the exponential time decay indicated in (3.20) and likewise in the stability statement (3.13).

5. The inflow problem. A stability statement similar to Theorem 3.1 is valid in the inflow case where $a<0$. Assume that the CFL condition (3.12) holds with $\eta_{0}=\eta_{0}(\beta, \alpha)$. Then (3.13) follows with discrete weights $\omega_{j}=$ $\left(1-x_{j}\right) w_{j} /\left(1+x_{j}\right)$ or $\omega_{j}=\left(1-x_{j}\right) w_{j}$.

The rest of this section is devoted to the proof of Theorem 3.1 according to the various cases outlined in the four lemmas below. We start with

Lemma 3.2 (Stability of the spectral tau method). Consider the spectral Jacobi tau method (3.4). Then Theorem 3.1 holds with

$$
\begin{gathered}
\omega_{j}=\frac{1+x_{j}}{1-x_{j}} w_{j}, \quad\left\{w_{j}=w_{j}^{G}(\alpha, \beta)\right\}_{j=1}^{N}=\text { Gauss-Jacobi weights, } \\
\eta_{0} \equiv \eta_{0}(\alpha, \beta)=\left\{\begin{array}{ll}
\frac{1}{2}(1+\beta), & \alpha+\beta \leq 0, \\
\frac{1}{2}(1-\alpha), & \alpha+\beta \geq 0,
\end{array} \quad \alpha, \beta \in(-1,1) .\right.
\end{gathered}
$$

Proof. Squaring of (3.7a) yields

$$
\begin{aligned}
\left\|v_{N}\left(\cdot, t^{m+1}\right)\right\|_{\omega}^{2}= & \left\|v_{N}\left(\cdot, t^{m}\right)\right\|_{\omega}^{2}+2 \Delta t \cdot a\left\langle v_{N}\left(\cdot, t^{m}\right), v_{N}^{\prime}\left(\cdot, t^{m}\right)\right\rangle \\
& +(\Delta t \cdot a)^{2}\left\|v_{N}^{\prime}\left(\cdot, t^{m}\right)\right\|_{\omega}^{2} \\
= & \left\|v_{N}\left(\cdot, t^{m}\right)\right\|_{\omega}^{2}+2 \Delta t \cdot a \mathrm{I}+(\Delta t \cdot a)^{2} \mathrm{II},
\end{aligned}
$$

and we turn to estimate the two expressions, I and II, on the right of (3.22).

First, let us note that since the $\pi_{N}$-polynomial $v_{N}\left(x, t^{m}\right)$ vanishes at the inflow boundary, $(3.3 \mathrm{~b})$, we have

$$
v_{N}\left(x, t^{m}\right)=(1-x) p(x) \text { for some } p(x) \equiv p_{N-1}(x) \in \pi_{N-1} .
$$


Also, a straightforward computation shows that

$$
\begin{aligned}
\left(w(x) \frac{1+x}{1-x}\right)^{\prime}(1-x)^{2} & =[(\beta-\alpha+2)-(\beta+\alpha) x] w(x) \\
& \geq 4 \eta_{0} w(x), \quad|x| \leq 1,
\end{aligned}
$$

where $\eta_{0}=\eta_{0}(\alpha, \beta)$ is given in $(3.21 \mathrm{~b})$.

Now, since $((1+x) /(1-x)) v_{N}\left(x, t^{m}\right) v_{N}^{\prime}\left(x, t^{m}\right) \in \pi_{2 N-1}$, the Gauss quadrature rule $(2.13)$ implies

$$
\begin{aligned}
\mathrm{I} & \equiv \sum_{j=1}^{N} w_{j} \frac{1+x_{j}}{1-x_{j}} v_{N}\left(x_{j}, t^{m}\right) v_{N}^{\prime}\left(x_{j}, t^{m}\right) \\
& =\int_{-1}^{1} w(x) \frac{1+x}{1-x} v_{N}\left(x, t^{m}\right) v_{N}^{\prime}\left(x, t^{m}\right) d x .
\end{aligned}
$$

We integrate by parts the right-hand side of $\mathrm{I}$, substitute $v_{N}\left(x, t^{m}\right)=(1-x) p(x)$ from (3.23), and in view of (3.24) we obtain

$$
\mathrm{I}=-\frac{1}{2} \int_{-1}^{1}\left(w(x) \frac{1+x}{1-x}\right)^{\prime}(1-x)^{2} p^{2}(x) d x \leq-2 \eta_{0}\|p\|_{w(x)}^{2}
$$

Next, let us consider the second expression, II, on the right of (3.22). As before, we substitute $v_{N}\left(x, t^{m}\right)=(1-x) p(x)$ from (3.23) and obtain

$$
\begin{aligned}
\mathrm{II} & \equiv\left\|v_{N}^{\prime}\left(\cdot, t^{m}\right)\right\|_{\omega}^{2}=\sum_{j=1}^{N} w_{j} \frac{1+x_{j}}{1-x_{j}}\left[\left(1-x_{j}\right) p^{\prime}\left(x_{j}\right)-p\left(x_{j}\right)\right]^{2} \\
& \leq 2 \sum_{j=1}^{N} w_{j}\left(1-x_{j}^{2}\right)\left(p^{\prime}\left(x_{j}\right)\right)^{2}+2 \sum_{j=1}^{N} w_{j} \frac{1+x_{j}}{1-x_{j}} p^{2}\left(x_{j}\right)=\mathrm{II}_{1}+\mathrm{II}_{2} .
\end{aligned}
$$

Since $\left(1-x^{2}\right)\left(p^{\prime}(x)\right)^{2} \in \pi_{2 N-2}$, the Gauss quadrature rule (2.13), followed by the inverse inequality (2.6), implies

$$
\mathrm{II}_{1} \equiv 2 \sum_{j=1}^{N} w_{j}\left(1-x_{j}^{2}\right)\left(p^{\prime}\left(x_{j}\right)\right)^{2}=2\left\|p^{\prime}\right\|_{\left(1-x^{2}\right) w(x)}^{2} \leq 2 \lambda_{N-1}\|p\|_{w(x)}^{2}, \quad p \in \pi_{N-1} \text {. }
$$

This, together with the obvious upper bound

$$
\mathrm{II}_{2} \equiv 2 \sum_{j=1}^{N} w_{j} \frac{1+x_{j}}{1-x_{j}} p^{2}\left(x_{j}\right) \leq \frac{4}{\Delta x_{\min }}\|p\|_{w(x)}^{2},
$$

gives us

$$
\mathrm{II} \leq\left(2 \lambda_{N-1}+\frac{4}{\Delta x_{\min }}\right)\|p\|_{w(x)}^{2} .
$$

Equipped with (3.25) and (3.26), we return to (3.22) to find

$$
\begin{aligned}
& \left\|v_{N}\left(\cdot, t^{m+1}\right)\right\|_{\omega}^{2} \\
& \quad \leq\left\|v_{N}\left(\cdot, t^{m}\right)\right\|_{\omega}^{2}-2 \Delta t \cdot a\left[2 \eta_{0}-\Delta t \cdot a\left(\lambda_{N-1}+\frac{2}{\Delta x_{\min }}\right)\right]\|p\|_{w(x)}^{2} .
\end{aligned}
$$


The CFL condition (3.12) and (3.21b) imply that the expression in square brackets on the right is positive,

$$
\left[2 \eta_{0}-\Delta t \cdot a\left(\lambda_{N-1}+\frac{2}{\Delta x_{\min }}\right)\right] \geq \eta_{0}>0,
$$

and hence strong stability holds.

In fact, one more application of Gauss quadrature yields

$$
\begin{aligned}
\|p\|_{w(x)}^{2} & =\sum_{j=1}^{N} w_{j} p^{2}\left(x_{j}\right)=\sum_{j=1}^{N} w_{j} \frac{v_{N}^{2}\left(x_{j}, t^{m}\right)}{\left(1-x_{j}\right)^{2}} \\
& \geq \sum_{j=1}^{N} w_{j} \frac{1+x_{j}}{1-x_{j}} v_{N}^{2}\left(x_{j}, t^{m}\right)=\left\|v_{N}\left(\cdot, t^{m}\right)\right\|_{\omega}^{2} .
\end{aligned}
$$

Inequalities (3.29), (3.28) together with (3.27) imply

$$
\left\|v_{N}\left(\cdot, t^{m+1}\right)\right\|_{\omega}^{2} \leq\left(1-2 \eta_{0} \Delta t \cdot a\right)\left\|v_{N}\left(\cdot, t^{m}\right)\right\|_{\omega}^{2},
$$

and the result (3.13) follows.

Next, we take advantage of the rather general setup we employed in Lemma 3.2. Specifically, since $P_{N+1}^{(\alpha, \beta)^{\prime}}$ is proportional to $P_{N}^{(\alpha+1, \beta+1)}$ (consult $(2.3)$ ), we may use Lemma 3.2 with $\eta_{0}(\alpha, \beta)$ replaced by $\eta_{0}(\alpha+1, \beta+1)$ to conclude:

Lemma 3.3 (Stability of the pseudospectral method). Consider the pseudospectral Jacobi method (3.5). Then Theorem 3.1 holds with

$$
\begin{aligned}
& \omega_{j}=\frac{1+x_{j}}{1-x_{j}} w_{j}, \\
& \quad\left\{w_{j}=w_{j}^{G}(\alpha+1, \beta+1)\right\}_{j=1}^{N}=\text { Gauss-Jacobi weights, } \\
& \quad \eta_{0} \equiv \eta_{0}(\alpha, \beta)=-\alpha / 2>0, \quad \alpha, \beta \in(-1,0) .
\end{aligned}
$$

As mentioned before, alternative proofs of Theorem 3.1 are possible. For example, following [6, Theorem 5.1], one may employ a stable norm weighted by $\omega_{j}=\left(1+x_{j}\right) w_{j}$ instead of the weights $\omega_{j}=\left(1+x_{j}\right) w_{j} /\left(1-x_{j}\right)$ used in (3.21a), (3.31a). We begin with

Lemma 3.4 (Stability of the spectral tau method revisited). Consider the spectral Jacobi tau method (3.4). Then Theorem 3.1 holds with

(3.32a) $\quad \omega_{j}=\left(1+x_{j}\right) w_{j}, \quad\left\{w_{j}=w_{j}^{G}(\alpha, \beta)\right\}_{j=1}^{N}=$ Gauss-Jacobi weights,

$$
\eta_{0}=\eta_{0}(\alpha, \beta)=\left\{\begin{array}{ll}
-\alpha / 2, & \alpha+\beta+1 \geq 0, \\
\frac{1}{2}(1+\beta), & \alpha+\beta+1 \leq 0,
\end{array} \quad \alpha, \beta \in(-1,0)\right.
$$


Proof. We square (3.7a), this time using the inner product weighted by (3.32a),

$$
\begin{aligned}
\left\|v_{N}\left(\cdot, t^{m+1}\right)\right\|_{\omega}^{2}= & \left\|v_{N}\left(\cdot, t^{m}\right)\right\|_{\omega}^{2}+2 \Delta t \cdot a\left\langle v_{N}\left(\cdot, t^{m}\right), v_{N}^{\prime}\left(\cdot, t^{m}\right)\right\rangle \\
& +(\Delta t \cdot a)^{2}\left\|v_{N}^{\prime}\left(\cdot, t^{m}\right)\right\|_{\omega}^{2} \\
= & \left\|v_{N}\left(\cdot, t^{m}\right)\right\|_{\omega}^{2}+2 \Delta t \cdot a \mathrm{I}+(\Delta t \cdot a)^{2} \mathrm{II}
\end{aligned}
$$

and as before we have to estimate the two expressions on the right of (3.33).

The first expression, I, involves discrete summation of

$$
f(x)=(1+x) v_{N}\left(x, t^{m}\right) v_{N}^{\prime}\left(x, t^{m}\right),
$$

and since $f(x)$ is a $\pi_{2 N}$-polynomial, its $N$-node Gaussian sum is not an exact integral. The essential observation here is that $f^{(2 N)} \equiv$ Const $>0$ in this case, and the error estimate $(2.14)$ tells us that the Gauss quadrature rule is upper bounded by

$$
\begin{aligned}
\mathrm{I} & \equiv \sum_{j=1}^{N} w_{j}\left(1+x_{j}\right) v_{N}\left(x_{j}, t^{m}\right) v_{N}^{\prime}\left(x_{j}, t^{m}\right) \\
& <\int_{-1}^{1} w(x)(1+x) v_{N}\left(x, t^{m}\right) v_{N}^{\prime}\left(x, t^{m}\right) d x .
\end{aligned}
$$

Let us recall that the homogeneous inflow boundary condition (3.7b) implies

$$
v_{N}\left(x, t^{m}\right)=(1-x) p(x) \text { for some } p(x) \equiv p_{N-1}(x) \in \pi_{N-1} .
$$

Also, a straightforward computation shows

$$
\begin{aligned}
(w(x)(1+x))^{\prime}(1-x) & =[(\beta-\alpha+1)-(\alpha+\beta+1) x] w(x) \\
& \geq 4 \eta_{0} w(x), \quad|x| \leq 1,
\end{aligned}
$$

where $\eta_{0}=\eta_{0}(\alpha, \beta)$ is given in $(3.32 \mathrm{~b})$.

We integrate by parts the right-hand side of I, substitute (3.35), and in view of (3.36) we obtain

$$
\mathrm{I}=-\frac{1}{2} \int_{-1}^{1}(w(x)(1+x))^{\prime}(1-x)^{2} p^{2}(x) d x \leq-2 \eta_{0}\|p\|_{(1-x) w(x)}^{2} .
$$

Next, let us consider the second expression, II, on the right of (3.33). As before, we substitute $v_{N}\left(x, t^{m}\right)=(1-x) p(x)$ from (3.35), and Gauss quadrature yields

$$
\begin{aligned}
\mathrm{II}= & \sum_{j=1}^{N} w_{j}\left(1+x_{j}\right)\left[\left(1-x_{j}\right) p^{\prime}\left(x_{j}\right)-p\left(x_{j}\right)\right]^{2} \\
= & \int_{-1}^{1} w(x)\left(1-x^{2}\right)(1-x)\left(p^{\prime}(x)\right)^{2} \\
& -2 \int_{-1}^{1} w(x)\left(1-x^{2}\right) p(x) p^{\prime}(x) d x+\int_{-1}^{1} w(x)(1+x) p^{2}(x) d x \\
= & \mathrm{II}_{1}+\mathrm{II}_{2}+\mathrm{II}_{3} .
\end{aligned}
$$


The inverse inequality (2.6) with weight $\omega(x)=(1-x) w(x)$ implies

$$
\mathrm{II}_{1}=\left\|p^{\prime}\right\|_{\left(1-x^{2}\right)(1-x) w(x)}^{2} \leq \lambda_{N-1}\|p\|_{(1-x) w(x)}^{2}, \quad \lambda_{N-1}=\lambda_{N-1}(\alpha+1, \beta),
$$

and integration by parts of $\mathrm{II}_{2}$ gives

$$
\begin{aligned}
\mathrm{II}_{2}+\mathrm{II}_{3} & =\int_{-1}^{1}\left[\left(w(x)\left(1-x^{2}\right)\right)^{\prime}+w(x)(1+x)\right] p^{2}(x) d x \\
& \leq 2\left\|p^{2}\right\|_{w(x)}=2 \sum_{j=1}^{N} w_{j} \frac{1-x_{j}}{1-x_{j}} p^{2}\left(x_{j}\right) \leq \frac{2}{\Delta x_{\min }}\|p\|_{(1-x) w(x)}^{2} .
\end{aligned}
$$

Consequently, we have

$$
\mathrm{II} \leq\left(\lambda_{N-1}+\frac{2}{\Delta x_{\min }}\right)\|p\|_{(1-x) w(x)}^{2} .
$$

Equipped with (3.37) and (3.39), we return to (3.33) to find

$$
\begin{aligned}
\left\|v_{N}\left(\cdot, t^{m+1}\right)\right\|_{\omega}^{2} \leq & \left\|v_{N}\left(\cdot, t^{m}\right)\right\|_{\omega}^{2}-2 \Delta t \\
& \cdot a\left[2 \eta_{0}-\Delta t \cdot \frac{a}{2}\left(\lambda_{N-1}+\frac{2}{\Delta x_{\min }}\right)\right]\|p\|_{(1-x) w(x)}^{2},
\end{aligned}
$$

and the result follows along the lines of Lemma 3.2 (consult (3.27)).

Lemma 3.4 does not cover the pseudospectral Jacobi methods, since by (2.3) the corresponding Jacobi parameters $\alpha+1, \beta+1 \notin(-1,0)$. However, the proof of Lemma 3.4 can be carried out in the pseudospectral case if we replace the Gauss quadrature rule by the Gauss-Lobatto one. We omit the almost identical details (which are outlined for the variable-coefficient case in Theorem 6.2 below) and state

Lemma 3.5 (Stability of the pseudospectral method revisited). Consider the pseudospectral Jacobi method (3.5). Then Theorem 3.1 holds with

$$
\omega_{j}=\left(1+x_{j}\right) w_{j}
$$

$$
\left\{w_{j}=w_{j}^{L}(\alpha, \beta)\right\}_{j=1}^{N}=\text { Gauss-Lobatto-Jacobi weights, }
$$

$$
\eta_{0}=\eta_{0}(\alpha, \beta)=\left\{\begin{array}{ll}
-\alpha / 2, & \alpha+\beta+1 \geq 0, \\
\frac{1}{2}(1+\beta), & \alpha+\beta+1 \leq 0,
\end{array} \quad \alpha, \beta \in(-1,0)\right.
$$

Remark. The stability asserted in Lemma 3.5 is stated in terms of the discrete seminorm $\left\|v_{N}(\cdot, t)\right\|_{\omega}^{2}=\sum_{j=1}^{N} w_{j}\left(1+x_{j}\right) v_{N}^{2}\left(x_{j}, t\right)$ weighted by the interior Gauss-Lobatto weights $\left\{w_{j}\right\}_{j=1}^{N}$. However, taking into account the homogeneous boundary condition (3.7b) and the exactness of Gauss-Lobatto quadrature for $\pi_{2 N+1}$-polynomials, this amounts to

$$
\left\|v_{N}(\cdot, t)\right\|_{\omega}^{2}=\sum_{j=0}^{N+1} w_{j}\left(1+x_{j}\right) v_{N}^{2}\left(x_{j}, t\right)=\int_{-1}^{1} w(x)(1+x) v_{N}^{2}(x, t) d x
$$


4. FORWARD EULER WITH INHOMOGENEOUS INITIAL-BOUNDARY CONDITIONS

We consider the inhomogeneous scalar hyperbolic equation

$$
u_{t}=a u_{x}+F(x, t), \quad(x, t) \in[-1,1] \times[0, \infty), a>0,
$$

which is augmented with inhomogeneous data prescribed at the inflow boundary

$$
u(1, t)=g(t), \quad t>0 .
$$

Using forward Euler time-differencing, the spectral approximation of (4.1) reads, at the $N$ zeros of $q_{N}(x)$,

$$
\begin{array}{r}
v_{N}\left(x_{j}, t^{m+1}\right)=v_{N}\left(x_{j}, t^{m}\right)+\Delta t \cdot a v_{N}^{\prime}\left(x_{j}, t^{m}\right)+\Delta t F\left(x_{j}, t^{m}\right), \\
q_{N}\left(x_{j}\right)=0,
\end{array}
$$

and is augmented with the boundary condition

$$
v_{N}\left(1, t^{m}\right)=g\left(t^{m}\right) \text {. }
$$

In this section, we study the stability of $(4.3 \mathrm{a}),(4.3 \mathrm{~b})$ in the two cases of

(4.4a) spectral Jacobi tau method: $q_{N}(x)=P_{N}^{(\alpha, \beta)}(x), \quad \alpha, \beta \in(-1,1)$, and the closely related

$$
\begin{array}{ll}
\text { pseudospectral Jacobi method : } & q_{N}(x)=P_{N+1}^{(\alpha, \beta)^{\prime}}(x), \\
& \alpha, \beta \in(-1,0) .
\end{array}
$$

To deal with the inhomogeneity of the boundary condition (4.3b), we employ a device introduced in $[6, \S 5]$. Namely, we consider the $\pi_{N}$-polynomial

$$
V_{N}(x, t)=v_{N}(x, t)-\frac{q_{N}(x)}{q_{N}(1)} g(t) .
$$

If we set

$$
\widetilde{F}(x, t)=F(x, t)+a \frac{q_{N}^{\prime}(x)}{q_{N}(1)} g(t),
$$

then $V_{N}(x, t)$ satisfies the inhomogeneous equation

$$
V_{N}\left(x_{j}, t^{m+1}\right)=V_{N}\left(x_{j}, t^{m}\right)+\Delta t \cdot a V_{N}^{\prime}\left(x_{j}, t^{m}\right)+\Delta t \widetilde{F}\left(x_{j}, t^{m}\right),
$$

which is now augmented by the homogeneous boundary condition

$$
V_{N}\left(1, t^{m}\right)=0 \text {. }
$$

Theorem 3.1 together with Duhamel's principle provide us with an a priori estimate of $\left\|V_{N}(\cdot, t)\right\|_{\omega}$ in terms of the initial and the inhomogeneous data, $\left\|V_{N}(\cdot, 0)\right\|_{\omega}$ and $\|\widetilde{F}(\cdot, t)\|_{\omega}$. Namely, if the CFL condition (3.12) holds, then we have

$$
\left\|V_{N}(\cdot, t)\right\|_{\omega} \leq e^{-\eta_{0} a t}\left\|V_{N}(\cdot, 0)\right\|_{\omega}+\sum_{0<t^{m} \leq t} \Delta t \cdot e^{-\eta_{0} a\left(t-t^{m}\right)}\left\|\widetilde{F}\left(\cdot, t^{m}\right)\right\|_{\omega} .
$$


Since the discrete norm $\|\cdot\|_{\omega}$ is supported at the zeros of $q_{N}(x)$, where $V_{N}\left(x_{j}, t\right)=v_{N}\left(x_{j}, t\right)$, we conclude

Theorem 4.1 (Stability of the spectral tau and pseudospectral Jacobi methods). Consider the spectral approximation (4.3a), (4.3b) associated with the Jacobi tau method (4.4a) or the pseudospectral Jacobi method (4.4b). There exists a positive constant $\eta_{0}=\eta_{0}(\alpha, \beta)>0$ independent of $N$, such that if the following CFL condition holds (consult (3.12)):

$$
\Delta t \cdot a\left(\lambda_{N-1}+\frac{2}{\Delta x_{\min }}\right) \leq \eta_{0}
$$

then the approximation (4.3a), (4.3b) satisfies the stability estimate

$$
\begin{aligned}
\left\|v_{N}(\cdot, t)\right\|_{\omega} \leq & e^{-\eta_{0} a t}\left\|v_{N}(\cdot, 0)\right\|_{\omega} \\
& +\sum_{0<t^{m} \leq t} \Delta t \cdot e^{-\eta_{0} a\left(t-t^{m}\right)} \\
& \cdot\left[\left\|F\left(\cdot, t^{m}\right)\right\|_{\omega}+a \frac{\left\|q_{N}^{\prime}(\cdot)\right\|_{\omega}}{\left|q_{N}(1)\right|}\left|g\left(t^{m}\right)\right|\right] .
\end{aligned}
$$

Theorem 4.1 provides us with an a priori stability estimate in terms of the initial data, $v_{N}(\cdot, 0)$, the inhomogeneous data, $F(\cdot, t)$, and the boundary data, $g(t)$. The dependence on the boundary data involves the factor of $\left\|q_{N}^{\prime}(\cdot)\right\|_{\omega} /\left|q_{N}(1)\right|$, which grows linearly with $N$, as shown by

Lemma 4.2. Let $\left\{w_{j}\right\}_{j=1}^{N}$ be the discrete weights given by either (3.32a) or (3.41a). Then there exists a constant independent of $N$ such that

$$
\frac{\left\|q_{N}^{\prime}(\cdot)\right\|_{\omega}}{\left|q_{N}(1)\right|} \leq \text { Const } \cdot N
$$

Remark. The stability estimate (4.10) together with (4.11) imply

$$
\begin{aligned}
& \left\|v_{N}(\cdot, t)\right\|_{\omega} \leq e^{-\eta_{0} a t}\left\|v_{N}(\cdot, 0)\right\|_{\omega} \\
& \quad+\sum_{0<t^{m} \leq t} \Delta t \cdot e^{-\eta_{0} a\left(t-t^{m}\right)} \cdot\left[\left\|F\left(\cdot, t^{m}\right)\right\|_{\omega}+\text { Const } \cdot N\left|g\left(t^{m}\right)\right|\right] .
\end{aligned}
$$

An inequality similar to (4.12) is encountered in the stability study of finite difference approximations to mixed initial-boundary hyperbolic systems [9]. We note in passing that the stability estimate (4.12) together with the usual consistency requirement guarantee the spectrally accurate convergence of the spectral approximation (consult [7] for the semidiscrete case).

Proof of Lemma 4.2. We consider, for example, the spectral tau method associated with $q_{N}(x)=P_{N}^{(\alpha, \beta)}(x)$ and with discrete weights $\omega_{j}=\left(1+x_{j}\right) w_{j}^{G}(\alpha, \beta)$. Using the Gauss and Gauss-Lobatto quadrature rules (2.13) and (2.16) in this order, we obtain

$$
\left\|q_{N}^{\prime}(\cdot)\right\|_{\omega}^{2}=\int_{-1}^{1} w(x)(1+x)\left(P_{N}^{(\alpha, \beta)^{\prime}}(x)\right)^{2} d x=2 w_{N+1}^{L}(\alpha, \beta)\left|P_{N}^{(\alpha, \beta)^{\prime}}(1)\right|^{2},
$$


and (4.11) follows in view of

$$
2 w_{N+1}^{L}(\alpha, \beta)\left|\frac{P_{N}^{(\alpha, \beta)^{\prime}}(1)}{P_{N}^{(\alpha, \beta)}(1)}\right|^{2} \leq \text { Const } \cdot N^{2} .
$$

Similar arguments (which are omitted) apply to the pseudospectral approximation associated with $q_{N}(x)=P_{N+1}^{(\alpha, \beta)^{\prime}}(x)$ and with discrete weights $\omega_{j}=$ $\left(1+x_{j}\right) w_{j}^{L}(\alpha, \beta)$.

\section{Multi-LeVel AND Runge-KutTA time-DifFerencing}

In the previous sections we proved the stability of spectral approximations which are combined with first-order accurate forward Euler time-differencing. In this section we extend our stability result for certain second- and third-order accurate multi-level and Runge-Kutta time-differencing, which were studied in $[10,11]$.

To this end, we view our $\pi_{N}$-approximate solution at time level $t, v(\cdot, t)$, as an $(N+1)$-dimensional column vector which is uniquely realized at the Gauss collocation nodes $\left(v\left(x_{1}, t\right), \ldots, v\left(x_{N}, t\right), v(1, t)\right)$.

The forward Euler time-differencing (3.7a) with homogeneous boundary conditions $(3.7 \mathrm{~b})$, reads

$$
v\left(t^{m}+\Delta t\right)=[\mathrm{I}+\Delta t \cdot a L] v\left(t^{m}\right), \quad a>0,
$$

where $L$ is an $(N+1) \times(N+1)$ matrix which accounts for the spatial spectral differencing together with the homogeneous boundary conditions,

$$
L v\left(t^{m}\right)=\left(v^{\prime}\left(x_{1}, t^{m}\right), \ldots, v^{\prime}\left(x_{N}, t^{m}\right), 0\right) .
$$

Remark. The construction of a spectral differentiation matrix $L$ can be accomplished in one of two ways. One possibility is carried out in the physical space, by exact differentiation of the unique $\pi_{N}$-interpolant which assumes the grid values $v\left(x_{1}, t\right), \ldots, v\left(x_{N}, t\right), v(1, t)$ at the corresponding Gauss nodes. This leads to full $(N+1) \times(N+1)$ differentiation matrices $L$, which are recorded for example in [2]. Spectral differencing in the physical space is then carried out by a matrix-vector multiplication at the expense of $O\left(N^{2}\right)$ operations. Alternatively, spectral differentiation can be accomplished in the transformed space. In the particular case of the Chebyshev (pseudo)spectral method, this leads to a factorization of the corresponding differentiation matrix $L$, whose matrixvector multiplication can be carried out efficiently by FFT requiring $O(N \log N)$ operations (consult $[8,2])$.

Theorem 3.1 tells us that if the CFL condition (3.12) holds, i.e., if

$$
\Delta t \cdot a\left(\lambda_{N-1}+\frac{2}{\Delta x_{\min }}\right) \leq \eta_{0},
$$

then $\mathrm{I}+\Delta t \cdot a L$ is bounded in the $\omega$-weighted induced operator norm,

$$
\|\mathrm{I}+\Delta t \cdot a L\|_{\omega} \leq e^{-\eta_{0} a \cdot \Delta t} .
$$


Let us consider an $(s+2)$-level time differencing method of the form

$$
\begin{aligned}
v\left(t^{m}+\Delta t\right)=\sum_{k=0}^{s} \theta_{k}\left[\mathrm{I}+c_{k} \Delta t \cdot a L\right] v\left(t^{m-k}\right) & \\
c_{k} & \geq 0, \theta_{k} \geq 0, \sum_{k=0}^{s} \theta_{k}=1 .
\end{aligned}
$$

In this case, $v\left(t^{m}+\Delta t\right)$ is given by a convex combination of stable forward Euler differencing, and we conclude

Corollary 5.1 (Multi-level time-differencing). Assume that the following CFL condition holds,

$$
\Delta t \cdot a\left(\lambda_{N-1}+\frac{2}{\Delta x_{\min }}\right) \leq \frac{\eta_{0}(\alpha, \beta)}{c_{k}}, \quad c_{k} \geq 0, k=0,1, \ldots, s .
$$

Then the spectral approximation (5.4) is strongly stable, and the following estimate holds:

$$
\left\|v_{N}(\cdot, t)\right\|_{\omega} \leq e^{-\eta_{*} a t}\left\|v_{N}(\cdot, 0)\right\|_{\omega}, \quad \eta_{*}=\min _{k} \frac{\eta_{0}}{c_{k}}>0 .
$$

In [10], second- and third-order accurate multi-level time-differencing methods of the positive type (5.4) were constructed. They take the particularly simple form

$$
v\left(t^{m}+\Delta t\right)=\theta\left[\mathrm{I}+c_{0} \Delta t \cdot a L\right] v\left(t^{m}\right)+(1-\theta)\left[\mathrm{I}+c_{s} \Delta t \cdot a L\right] v\left(t^{m-s}\right),
$$

with positive coefficients given in Table 1 .

TABLE 1

Multi-level methods

\begin{tabular}{|l|c|c|c|}
\hline Second-order time-differencing & $\theta$ & $c_{0}$ & $c_{s}$ \\
\hline 4-level method $(s=2)$ & $\frac{3}{4}$ & 2 & 0 \\
5-level method $(s=3)$ & $\frac{8}{9}$ & $\frac{3}{2}$ & 0 \\
\hline Third-order time-differencing & & & \\
\hline 5-level method $(s=3)$ & $\frac{16}{27}$ & 3 & $\frac{12}{11}$ \\
6-level method $(s=4)$ & $\frac{25}{32}$ & 2 & $\frac{10}{7}$ \\
7-level method $(s=5)$ & $\frac{108}{125}$ & $\frac{5}{3}$ & $\frac{30}{17}$ \\
\hline
\end{tabular}


Similar arguments apply for Runge-Kutta time-differencing methods. In this case the resulting positive-type Runge-Kutta methods take the form

$$
\begin{gathered}
v^{(1)}\left(t^{m+1}\right)=[\mathrm{I}+\Delta t \cdot a L] v\left(t^{m}\right), \\
v^{(k)}\left(t^{m+1}\right)=\theta_{k} v\left(t^{m}\right)+\left(1-\theta_{k}\right)[\mathrm{I}+\Delta t a L] \cdot v^{(k-1)}\left(t^{m+1}\right), \\
k=2, \ldots, s, \\
v\left(t^{m+1}\right)=v^{(s)}\left(t^{m+1}\right) .
\end{gathered}
$$

We state

Corollary 5.2 (Runge-Kutta time-differencing). Assume that the CFL condition (3.12) holds. Then the spectral approximation (5.8a-c) with $0 \leq \theta_{k}<1$ is strongly stable, and the stability estimate (3.13) holds.

In Table 2 we quote the preferred second- and third-order choices of [11].

TABLE 2

Runge-Kutta methods

\begin{tabular}{|l|c|c|}
\hline Second-order time-differencing & $\theta_{2}$ & $\theta_{3}$ \\
\hline Two-step modified Euler $(s=2)$ & $\frac{1}{2}$ & - \\
\hline Third-order time-differencing & & \\
\hline Three-step method $(s=3)$ & $\frac{3}{4}$ & $\frac{1}{3}$ \\
\hline
\end{tabular}

Remarks. 1. The above results can be extended to include nonhomogeneous data as well. We omit the details.

2. In $[10,11]$, higher-order accurate $(>3)$ multi-level and Runge-Kutta timedifferencing were constructed. In the present context (of constant-coefficient spectral approximations), they amount to convex combinations of the stable forward Euler differencing $\mathrm{I}+\Delta t \cdot a L$ and its adjoint $\mathrm{I}-\Delta t \cdot a L$. The above argument does not cover these cases, however, since in our case the stability of $\mathrm{I} \pm \Delta t \cdot a L$ is measured by different weighted norms.

\section{SCALAR EQUATIONS WITH VARIABLE COEFFICIENTS}

We begin with

Epilogue. When dealing with finite difference approximations which are locally supported, i.e., finite difference schemes whose stencils occupy a finite number of neighboring grid cells, each of which is of size $\Delta x$, one then encounters the hyperbolic CFL stability restriction

$$
\frac{\Delta t}{\Delta x}|a| \leq \text { Const } .
$$


With this in mind, it is tempting to provide a heuristic justification for the stability of spectral methods, by arguing that a CFL stability restriction similar to (6.1) should hold. Namely, when $\Delta x$ is replaced by the minimal grid size, $\Delta x_{\min }=\min _{j}\left|x_{j+1}-x_{j}\right|=O\left(N^{-2}\right)$, then (6.1) leads to

$$
\Delta t \cdot|a| N^{2} \leq \text { Const } .
$$

Although the final conclusion is correct (consult (3.16)), it is important to realize that this "handwaving" argument is not well founded in the case of spectral methods. Indeed, since the spectral stencils occupy the whole interval $(-1,1)$, spectral methods do not lend themselves to the stability analysis of locally supported finite difference approximations. Of course, by the same token, this explains the existence of unconditionally stable fully implicit (and hence globally supported) finite difference approximations.

As noted earlier, our stability proof (in Theorem 3.1) shows that the CFL condition (6.2) is related to the following two points:

\#1. The size of the corresponding Sturm-Liouville eigenvalues, $\lambda_{N-1}=$ $O\left(N^{2}\right)$.

\#2. The minimal grid size, $1 / \Delta x_{\min }=O\left(N^{2}\right)$.

The second point seems to support the fact that $\Delta x_{\text {min }}$ plays an essential role in the CFL stability restriction for the global spectral methods, as predicted by the local heuristic argument outlined above. To clarify this issue, we study in this section the stability of spectral approximations to scalar hyperbolic equations with variable coefficients. The principal raison d'être, which motivates our present study, is to show that our stability analysis in the constant-coefficient case is versatile enough to deal with certain variable-coefficient problems.

We begin with the particular example introduced in [8],

$$
u_{t}=-x u_{x}, \quad(x, t) \in[-1,1] \times[0, \infty) .
$$

We shall show that the CFL stability restriction in this case is related to the $O\left(N^{2}\right)$-size of the Sturm-Liouville eigenvalues (point \#1 above), but otherwise it is independent of the minimal grid size mentioned in point \#2 above.

Observe that (6.3) requires no augmenting boundary conditions, since both boundaries, $x= \pm 1$, are outflow ones. Consequently, the various $\pi_{N}$-spectral approximations of (6.3) with forward Euler time-differencing read

$$
v_{N}\left(x, t^{m}+\Delta t\right)=v_{N}\left(x, t^{m}\right)-\Delta t \cdot x v_{N}^{\prime}\left(x, t^{m}\right) .
$$

We have

Theorem 6.1 (Stability). Assume that the following CFL condition holds:

$$
\Delta t \cdot \lambda_{N} \leq 1, \quad \lambda_{N}=N(N+1) .
$$

Then the spectral approximation (6.4) is stable, and the following estimate is fulfilled:

$$
\left\|v_{N}(\cdot, t)\right\|_{1-x^{2}} \leq e^{t}\left\|v_{N}(\cdot, 0)\right\|_{1-x^{2}} .
$$


Proof. Squaring (6.4) yields

$$
\begin{aligned}
\left\|v_{N}\left(\cdot, t^{m+1}\right)\right\|_{1-x^{2}}^{2}= & \left\|v_{N}\left(\cdot, t^{m}\right)\right\|_{1-x^{2}}^{2}-2 \Delta t\left(v_{N}\left(\cdot, t^{m}\right), x v_{N}^{\prime}\left(\cdot, t^{m}\right)\right)_{1-x^{2}} \\
& +(\Delta t)^{2}\left\|x v_{N}^{\prime}\left(\cdot, t^{m}\right)\right\|_{1-x^{2}}^{2} \\
= & \left\|v_{N}\left(\cdot, t^{m}\right)\right\|_{1-x^{2}}^{2}+2 \Delta t \cdot \mathrm{I}+(\Delta t)^{2} \cdot \mathrm{II} .
\end{aligned}
$$

Integration by parts shows that the first expression, I, equals

$$
\begin{aligned}
\mathrm{I} & \equiv \frac{1}{2} \int_{-1}^{1}\left(x\left(1-x^{2}\right)\right)^{\prime} v_{N}^{2}\left(x, t^{m}\right) d x \\
& =\frac{1}{2}\left\|v_{N}\left(\cdot, t^{m}\right)\right\|_{1-x^{2}}^{2}-\int_{-1}^{1} x^{2} v_{N}^{2}\left(x, t^{m}\right) d x .
\end{aligned}
$$

Next, we write $x v_{N}^{\prime} \equiv\left(x v_{N}\right)^{\prime}-v_{N}$, and by the inverse inequality (2.6) the second expression, II, can be bounded as

$$
\begin{aligned}
\mathrm{II} & =\left\|\left(x v_{N}\left(x, t^{m}\right)\right)^{\prime}\right\|_{1-x^{2}}^{2}-2 \int_{-1}^{1} x^{2} v_{N}^{2}\left(x, t^{m}\right) d x \\
& \leq\left(\lambda_{N}-2\right) \int_{-1}^{1} x^{2} v_{N}^{2}\left(x, t^{m}\right) d x .
\end{aligned}
$$

Inserting (6.8) and (6.9) into (6.7), we end up with

$$
\begin{aligned}
\left\|v_{N}\left(\cdot, t^{m+1}\right)\right\|_{1-x^{2}}^{2} \leq & (1+\Delta t) \cdot\left\|v_{N}\left(\cdot, t^{m}\right)\right\|_{1-x^{2}}^{2} \\
& +\Delta t \cdot\left[\left(\lambda_{N}-2\right) \Delta t-2\right] \cdot \int_{-1}^{1} x^{2} v_{N}^{2}(x, t) d x .
\end{aligned}
$$

The CFL condition (6.5) tells us that the contribution of the second term is negative, and the stability estimate (6.6) now follows.

We now turn to discuss scalar hyperbolic equations with positive variable coefficients,

$$
u_{t}=a(x) u_{x}, \quad 0<a(x)<a_{\infty}, \quad(x, t) \in[-1,1] \times[0, \infty),
$$

which are augmented with homogeneous conditions at the inflow boundary

$$
u(1, t)=0 .
$$

We consider the pseudospectral Jacobi method collocated at the $N$ zeros of $P_{N+1}^{(\alpha, \beta)^{\prime}}(x)$. Using forward Euler time-differencing, the resulting approximation reads

(6.13a) $v_{N}\left(x_{j}, t^{m+1}\right)=v_{N}\left(x_{j}, t^{m}\right)+\Delta t \cdot a\left(x_{j}\right) v_{N}^{\prime}\left(x_{j}, t^{m}\right), \quad P_{N+1}^{(\alpha, \beta)}\left(x_{j}\right)=0$, together with the boundary condition

$$
v_{N}\left(1, t^{m}\right)=0 \text {. }
$$


Arguing along the lines of Theorem 3.1, we have

Theorem 6.2 (Stability of the pseudospectral Jacobi method with variable coefficients). Consider the pseudospectral Jacobi approximation (6.13a), (6.13b). There exists a constant $\eta_{0} \equiv \eta_{0}(\alpha, \beta)$,

(6.14a) $\eta_{0} \equiv \eta_{0}(\alpha, \beta)=\left\{\begin{array}{ll}-\alpha / 2, & \alpha+\beta+1 \geq 0, \\ \frac{1}{2}(1+\beta), & \alpha+\beta+1 \leq 0,\end{array} \quad \alpha, \beta \in(-1,0)\right.$,

such that if the following CFL condition holds:

$$
\Delta t\left(a_{\infty} \lambda_{N-1}+2 \max _{1 \leq j \leq N} \frac{a\left(x_{j}\right)}{1-x_{j}}\right) \leq \eta_{0}
$$

then the approximation (6.13a), (6.13b) is strongly stable, i.e., there exist discrete weights

$$
\begin{aligned}
\omega_{j}=\left(1+x_{j}\right) \frac{w_{j}}{a\left(x_{j}\right)}, & \\
& \left\{w_{j}=w_{j}^{L}(\alpha, \beta)\right\}_{j=1}^{N}=\text { Gauss-Lobatto weights, }
\end{aligned}
$$

such that

$$
\left\|v_{N}(\cdot, t)\right\|_{\omega} \leq\left\|v_{N}(\cdot, 0)\right\|_{\omega} .
$$

Proof. We divide $(6.13 \mathrm{a})$ by $\sqrt{a\left(x_{j}\right)}$,

$$
\frac{1}{\sqrt{a\left(x_{j}\right)}} v_{N}\left(x_{j}, t^{m+1}\right)=\frac{1}{\sqrt{a\left(x_{j}\right)}} v_{N}\left(x_{j}, t^{m}\right)+\Delta t \cdot \sqrt{a\left(x_{j}\right)} \cdot v_{N}^{\prime}\left(x_{j}, t^{m}\right),
$$

and, proceeding as before, we square both sides to obtain

$$
\begin{aligned}
\left\|v_{N}\left(\cdot, t^{m+1}\right)\right\|_{\omega}^{2}= & \left\|v_{N}\left(\cdot, t^{m}\right)\right\|_{\omega}^{2}+2 \Delta t\left\langle v_{N}\left(\cdot, t^{m}\right), v_{N}^{\prime}\left(\cdot, t^{m}\right)\right\rangle \\
& +(\Delta t)^{2}\left\|a(\cdot) v_{N}^{\prime}\left(\cdot, t^{m}\right)\right\|_{\omega}^{2} \\
= & \left\|v_{N}\left(\cdot, t^{m}\right)\right\|_{\omega}^{2}+2 \Delta t \cdot \mathrm{I}+(\Delta t)^{2} \cdot \mathrm{II} .
\end{aligned}
$$

The first expression, I, involves discrete summation of the $\pi_{2 N}$-polynomial $f(x)=(1+x) v_{N}\left(x, t^{m}\right) v_{N}^{\prime}\left(x, t^{m}\right)$, and since $f( \pm 1)=0$ (in view of $(6.13 \mathrm{~b})$ ), the $N$-node Gauss-Lobatto quadrature rule yields

$$
\begin{aligned}
\mathrm{I} & \equiv \sum_{j=0}^{N+1} w_{j}^{L}\left(1+x_{j}\right) v_{N}\left(x_{j}, t^{m}\right) v_{N}^{\prime}\left(x_{j}, t^{m}\right) \\
& =\int_{-1}^{1} w(x)(1+x) v_{N}\left(x, t^{m}\right) v_{N}^{\prime}\left(x, t^{m}\right) d x .
\end{aligned}
$$


We integrate by parts the right-hand side of $\mathrm{I}$, substitute $v_{N}\left(x, t^{m}\right)=(1-x) p(x)$ from (3.35), and in view of (3.36) we obtain as before (compare (3.37)),

$$
\mathrm{I} \leq-2 \eta_{0}\|p\|_{(1-x) w(x)}^{2} .
$$

The second expression, II, gives us

$$
\begin{aligned}
\mathrm{II}= & \sum_{j=1}^{N} w_{j} a\left(x_{j}\right)\left(1+x_{j}\right)\left[\left(1-x_{j}\right) p^{\prime}\left(x_{j}\right)-p\left(x_{j}\right)\right]^{2} \\
\leq & 2 a_{\infty} \sum_{j=1}^{N} w_{j}\left(1-x_{j}^{2}\right)\left(1-x_{j}\right)\left(p^{\prime}\left(x_{j}\right)\right)^{2} \\
& +2 \sum_{j=1}^{N} w_{j} a\left(x_{j}\right)\left(1+x_{j}\right) p^{2}\left(x_{j}\right) \\
= & 2 a_{\infty} \mathrm{II}_{1}+2 \cdot \mathrm{II}_{2} .
\end{aligned}
$$

The inverse inequality (2.6) with weight $\omega(x)=(1-x) w(x)$ implies

$$
\mathrm{II}_{1}=\left\|p^{\prime}\right\|_{\left(1-x^{2}\right)(1-x) w(x)}^{2} \leq \lambda_{N-1}\|p\|_{(1-x) w(x)}^{2}, \quad \lambda_{N-1}=\lambda_{N-1}(\alpha+1, \beta),
$$

and the expression $\mathrm{II}_{2}$ can be bounded as

$$
\begin{aligned}
\mathrm{II}_{2} & \leq \max _{1 \leq j \leq N}\left[a\left(x_{j}\right) \frac{1+x_{j}}{1-x_{j}}\right] \cdot \sum_{j=0}^{N+1} w_{j}\left(1-x_{j}\right) p^{2}\left(x_{j}\right) \\
& \leq 2 \cdot \max _{1 \leq j \leq N} \frac{a\left(x_{j}\right)}{1-x_{j}} \cdot\|p\|_{(1-x) w(x)}^{2} .
\end{aligned}
$$

Consequently, we have

$$
\mathrm{II} \leq 2\left(a_{\infty} \lambda_{N-1}+2 \cdot \max _{1 \leq j \leq N} \frac{a\left(x_{j}\right)}{1-x_{j}}\right)\|p\|_{(1-x) w(x)}^{2} .
$$

Equipped with (6.17) and (6.19) we return to (6.16) to find

$$
\begin{aligned}
\left\|v_{N}\left(\cdot, t^{m+1}\right)\right\|_{\omega}^{2} & \\
\leq\left\|v_{N}\left(\cdot, t^{m}\right)\right\|_{\omega}^{2}-2 \Delta t\left[2 \eta_{0}-\Delta t\right. & \left(a_{\infty} \lambda_{N-1}\right. \\
& \left.\left.+2 \max _{1 \leq j \leq N} \frac{a\left(x_{j}\right)}{1-x_{j}}\right)\right]\|p\|_{(1-x) w(x)}^{2},
\end{aligned}
$$

and $(6.15 b)$ follows in view of the CFL condition (6.14b).

Notes. 1 . The case $a\left(x_{j}\right) \equiv a=$ Const $>0$ corresponds to the stability statement of Lemma 3.5. Similar stability statements with the appropriate weights which correspond to Lemmas 3.2, 3.3, and 3.4, namely,

$$
\omega_{j}=\left(\left(1+x_{j}\right) /\left(1-x_{j}\right)\right) w_{j}^{G} / a\left(x_{j}\right) \text { and } \omega_{j}=\left(1+x_{j}\right) w_{j}^{G} / a\left(x_{j}\right),
$$

hold. These statements cover the stability of the corresponding spectral and pseudospectral Jacobi approximations with variable coefficients. 
2. We should highlight the fact that the stability assertion stated in Theorem 6.2 depends solely on the uniform bound of $a\left(x_{j}\right)$, but otherwise is independent of the smoothness of $a(x)$.

3. The proof of Theorem 6.2 applies mutatis mutandis to the case of variable coefficients with $a=a(x, t)$. If $a\left(x_{j}, t\right)$ are $C^{1}$-functions in the time variable, then $(6.20)$ is replaced by

$\left\|v_{N}\left(\cdot, t^{m+1}\right)\right\|_{\omega^{m+1}} \leq(1+$ Const $\cdot \Delta t)\left\|v\left(\cdot, t^{m}\right)\right\|_{\omega^{m}}$,

$$
\omega_{j}^{m}=\left(1+x_{j}\right) \frac{w_{j}^{L}}{a\left(x_{j}, t^{m}\right)},
$$

and stability follows.

4. We conclude by noting that the CFL condition (6.14b) depends on the quantity $\max _{1 \leq j \leq N} a\left(x_{j}\right) /\left(1-x_{j}\right)$, rather than the minimal grid size, $1 / \Delta x_{\min }$, as in the constant-coefficient case (compare (3.12)). This amplifies our introductory remarks at the beginning of this section, which claim that the $O\left(N^{-2}\right)$ stability restriction is essentially due to the size of the Sturm-Liouville eigenvalues, $\lambda_{N-1}=O\left(N^{2}\right)$. Indeed, the other portion of the CFL condition, requiring

$$
\Delta t \cdot 2 \max _{1 \leq j \leq N} \frac{a\left(x_{j}\right)}{1-x_{j}} \leq \eta_{0},
$$

guarantees the resolution of waves entering through the inflow boundary $x=1$. In the constant-coefficient case this resolution requires time steps $\Delta t$ of size $1 / \Delta x_{\min }$. However, when the inflow boundary is almost characteristic, i.e., when $a(1) \sim 0$, then the CFL condition is essentially independent of $\Delta x_{\min }$, for $(6.21)$ boils down to $\Delta t \cdot 2 a^{\prime}(1) \leq \eta_{0}$. In purely outflow cases such as $(6.3)$, the time step is independent of any resolution requirement at the boundaries, and we are left with the CFL condition (6.5) stated in Theorem 6.1.

\section{ACKNOWLEDGMENT}

We thank Professor Walter Gautschi for carefully reading the first version of this paper and for making several helpful corrections.

\section{BIBLIOGRAPHY}

1. M. Abramowitz and I. A. Stegun, Handbook of mathematical functions with formulas, graphs, and mathematical tables, Government Printing Office, Washington, D.C., 1972.

2. C. Canuto, M. Y. Hussaini, A. Quarteroni, and T. Zang, Spectral methods in fluid dynamics, Springer-Verlag, New York, 1988.

3. C. Canuto and A. Quarteroni, Approximation results for orthogonal polynomials in Sobolev spaces, Math. Comp. 38 (1982), 67-86.

4. P. J. Davis and P. Rabinowitz, Methods of numerical integration, 2nd ed., Academic Press, New York, 1984.

5. D. Gottlieb, The stability of pseudospectral Chebyshev methods, Math. Comp. 36 (1981), 107-118.

6. D. Gottlieb, L. Lustman, and E. Tadmor, Stability analysis of spectral methods for hyperbolic initial-boundary value systems, SIAM J. Numer. Anal. 24 (1987), 241-256. 
7. D. Gottlieb, L. Lustman, and E. Tadmor, Convergence of spectral methods for hyperbolic initial-boundary value systems, SIAM J. Numer. Anal. 24 (1987), 532-537.

8. D. Gottlieb and S. Orszag, Numerical analysis of spectral methods: Theory and applications, SIAM, Philadelphia, PA, 1977.

9. B. Gustafsson, H. O. Kreiss, and A. Sundström, Stability theory of difference approximations for mixed initial boundary value problems. II, Math. Comp. 26 (1972), 649-688.

10. C. W. Shu, Total-variation-diminishing time discretizations, SIAM J. Sci. Statist. Comput. 6 (1988), 1073-1084.

11. C. W. Shu and S. Osher, Efficient implementation of essentially non-oscillatory shock-capturing schemes, J. Comput. Phys. 77 (1988), 439-471.

12. A. H. Stroud and D. Secrest, Gaussian quadrature formulas, Prentice-Hall, Englewood Cliffs, N. J., 1966.

13. G. Szegö, Orthogonal polynomials, 4th ed., Amer. Math. Soc., Providence, R. I., 1975.

(D. Gottlieb and E. Tadmor) School of Mathematical Sciences, Tel-Aviv University, TEL-Aviv 69978, ISRAEL

(D. Gottlieb) Division of Applied Mathematics, Brown University, Providence, Rhode ISLAND 02912

(E. Tadmor) Department of Mathematics, University of Michigan, ANn Arbor, MichiGAN 48109

E-mail address: tadmor@math.lsa.umich.edu 